\title{
Spontaneous Intraparenchymal Lung Haematoma with Active Bleeding, Associated with S. Aureus
}

\author{
Riccardo Gerloni ${ }^{1}$, Nicola Artusi ${ }^{1}$, Stefano Cernic ${ }^{2}$
}

${ }^{1}$ Emergency Medicine Department, Azienda Sanitaria Universitaria Integrata di Trieste, ${ }^{2}$ Radiology Department, Azienda Sanitaria Universitaria Integrata di Trieste

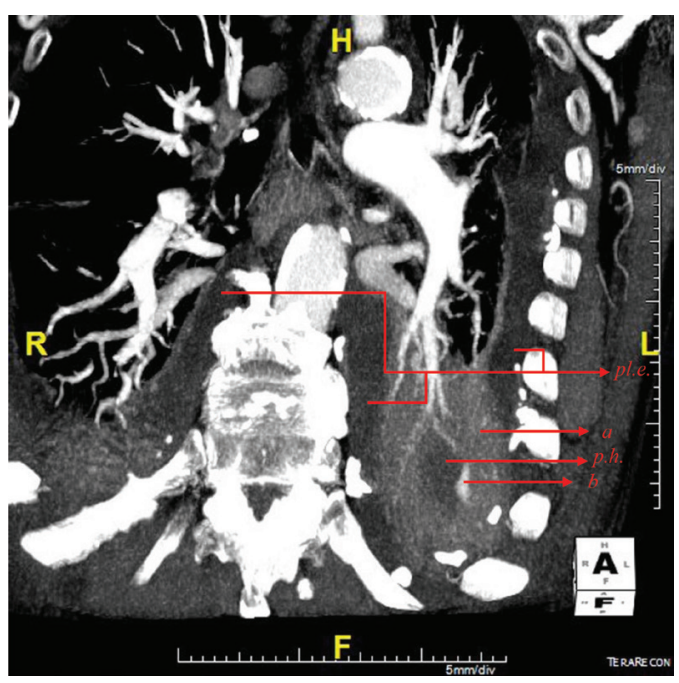

Figure 1. Oblique inferior coronal projection. $\mathrm{a}=$ Atelectasis; $\mathrm{b}=$ Active bleeding; $\mathrm{p} . \mathrm{h} .=$ Pulmonary hematoma; pl.e.=Pleural effusion.

A 91-year-old patient with hypertension and chronic kidney impairment was evaluated for dyspnoea and haemoptysis in a peripheral hospital. The patient appeared pale, dyspnoeic, and tachycardic, but normotensive, presenting with a vesicular murmur reduction in the lower left pulmonary field, normocytic anaemia (haemoglobin $8.6 \mathrm{~g} /$ $\mathrm{dL})$, white blood cell count: $5.68 \times 10^{\wedge} 3 /$ $\mu \mathrm{L}, \mathrm{C}$-reactive protein: $26.4 \mathrm{mg} / \mathrm{L}$, moderate renal impairment and normal coagulation tests (INR 1.15, APTT 27.9 s). A left

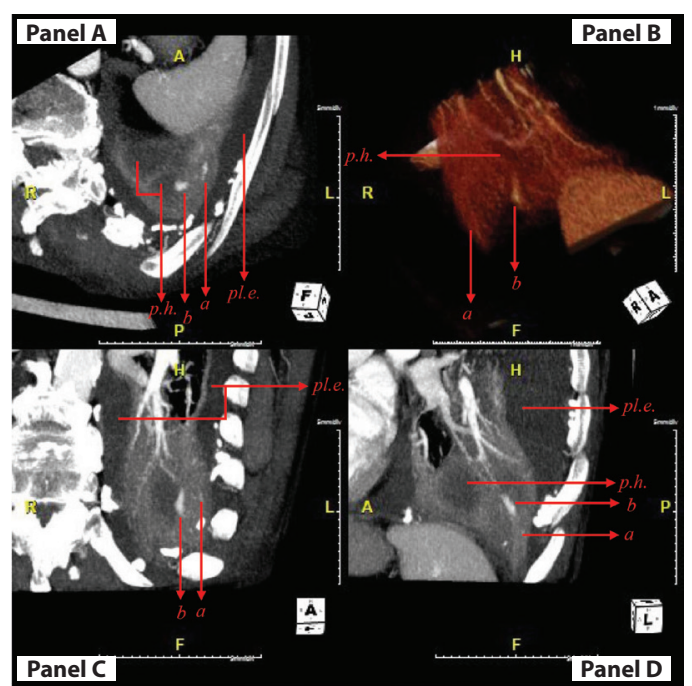

Figure 2. Comparison of oblique projections. Panel A: oblique left posterior transverse planes; Panel B: oblique right inferior coronal plane. 3D-volumerendered vascular distribution in left lower lobe CT-angiography; Panel C: oblique inferior coronal projection. Lower left lobe detail; Panel D: oblique anterior superior left sagittal plane.

lower lobe pulmonary hematoma $(4.8 \mathrm{~cm})$ with active bleeding (Figure 1) and pleural effusion with partial atelectasis were found on the chest CT-angiography (Figure 2). Further, some enlarged mediastinal lymph nodes were evident, as well as diffuse emphysema [not shown, $A / N$ ]. The patient was transfused and sent to a hospital with inter- 
ventional radiology; however neither embolization nor thoracic surgery were attempted because of the peripheral localization of the bleeding and the patient's clinical features. Conservative treatment and antibiotics permitted clinical and laboratory improvement. Quantiferon and the Venereal Disease Research Laboratory excluded tuberculosis or T. pallidum infection; a bronchoscopy with bronchoalveolar lavage and transbronchial needle aspiration was performed, showing no evidence of malignancy; neutrophils and positive culture tests suggested acute $S$. aureus respiratory infection. The diagnosis of spontaneous pulmonary hematoma with intraparenchymal haemorrhage was made, as no history of trauma was reported and we could not find any known risk factors. Parenchymal lung hematomas presenting with haemorrhage are rare, life-threatening conditions, associated with trauma, chest surgery, cancer, tuberculosis, vascular malformations and anticoagulation. Only a few cases of spontaneous idiopathic pulmonary hematomas have been reported, all showing parenchymal lung disorders, such as chronic obstructive pulmonary disease (1), isolated bronchiectasis (2) or emphysema (3), suggesting that primary lung diseases may underlie spontaneous pulmonary haemorrhage. However, all these patients had some possible risk factors, such as the use of platelet aggregation inhibition therapy or steroid drugs. None of these conditions was present in our patient. Probably the precipitating factor in our case of parenchymal lung haemorrhage was bronchitis, although it has never been reported as an isolated risk factor for spontaneous intraparenchymal lung hematoma with haemorrhage.

Key words: Lung haemorrhage - Lung bleeding Haemoptysis.

Acknowledgment: We would like to thank the Radiology Department, Ospedale di Cattinara, ASUITS Trieste, for providing the images.

Authors' contributions: Conception and design: RG and NA; Acquisition, analysis and interpretation of data: RG, NA and SC; Drafting the article: RG and NA; Revising it critically for important intellectual content: RG and NA; Approved final version of the manuscript: RG, NA and SC.

Conflict of interest: The authors declare that they have no conflict of interest.

Correspondence: nicola.artusi.1@gmail.com

Tel.: + 393384499930

Fax.: + 390403994060

Received: 15 February 2018

Accepted: 31 May 2018

\section{References}

1. Withey S, Tamimi A. Spontaneous pulmonary haemorrhage into an existing emphysematous bulla. BMJ Case Rep. 2016;2016:bcr2015213144.

2. Antón E, Loperena J, Lejarcegui JT. A 75-year-old woman with a lung nodule--a spontaneous pulmonary haematoma associated with bronchiectasis. Age Ageing. 2005;34(3):310-1.

3. Jay SJ, Johanson G. Massive intrapulmonary hemorrhage: an uncommon complication of bullous emphysema. Am Rev Respir Dis. 1974;110(4):497501. 